\title{
The problem of kernel determination from viscoelasticity system integro-differential equations for homogeneous anisotropic media
}

\author{
D. K. Durdiev ${ }^{1}$, U. D. Durdiev ${ }^{2}$ \\ ${ }^{1}$ Bukhara State University, Bukhara, Uzbekistan \\ ${ }^{2}$ Kazan Federal University, Kazan, Russia \\ durdiev65@mail.ru, umidjan93@mail.ru
}

\begin{abstract}
We consider the problem of reconstructing the time-dependent history of the viscoelasticity medium from the viscoelasticity system of equations for an homogeneous anisotropic medium. As additional information, the Fourier image of the displacement vector for values $\nu=\nu_{0} \neq 0$ of transformation parameter is given. It is shown that if the given functions satisfy some conditions of agreement and smoothness, the solution for the posed problem is uniquely defined in the class of a continuous functions and it continuously depends on given functions.
\end{abstract}

Keywords: inverse problem, integro-differential equation, delta function, Fourier transformation, agreement condition.

Received: 8 March 2016

Revised: 20 April 2016

\section{Setting up the problem and main result}

We consider the integro-differential system for $x=\left(x_{1}, x_{2}, x_{3}\right) \in R^{3}, t \in R$ :

$$
\rho \frac{\partial^{2} u_{i}}{\partial t^{2}}=\sum_{j=1}^{3} \frac{\partial T_{i j}}{\partial x_{j}}+f(x, t), i=1,2,3,
$$

at the initial conditions

$$
\left.u_{i}\right|_{t<0} \equiv 0 .
$$

Here, $u(x, t)=\left(u_{1}(x, t), u_{2}(x, t), u_{3}(x, t)\right)^{*}$ is the displacement vector function, $*$ is the sign of transposition, $T_{i j}$ denotes the stress tensor related to the viscoelastic medium. More exactly, we have:

$$
T_{i j}(x, t)=\sum_{k, l=1}^{3} c_{i j k l}\left\{\frac{\partial u_{k}}{\partial x_{l}}(x, t)+\int_{0}^{t} K_{i}(\tau) \frac{\partial u_{k}}{\partial x_{l}}(x, t-\tau) d \tau\right\},
$$

$f(x, t)=\left(f_{1}(x, t), f_{2}(x, t), f_{3}(x, t)\right)^{*}$ is the external force; $\rho>0$ is the density of the medium. In equality (3), coefficients $c_{i j k l}$ are the elastic moduli of the medium. It is convenient and customary to describe the elastic moduli in the terms of a $6 \times 6$ matrix according to the following conventions relating a pair $(i, j)$ of indices $i, j=1,2,3$ to a single index $\alpha=1,2, \ldots, 6:(11) \rightarrow 1,(22) \rightarrow 2,(33) \rightarrow 3,(23)=(32) \rightarrow 4,(13)=(31) \rightarrow 5$, $(12)=(21) \rightarrow 6$. This correspondence is possible due to the symmetry properties $c_{i j k l}=c_{j i k l}=c_{i j l k}$. The additional symmetry property $c_{i j k l}=c_{k l i j}$ implies that the matrix $C=\left(c_{\alpha \beta}\right)_{6 \times 6}$ of all moduli is symmetric, where $\alpha=(i j), \beta=(k l)$. We will also assume that $\rho>0, c_{i j k l}$ are constants and the matrix $C=\left(c_{\alpha \beta}\right)_{6 \times 6}$ is positive definite.

Many important materials used in modern technologies (such as nanotechnology) are viscoelastic and anisotropic. Viscoelastic materials have the properties of viscosity and elasticity upon deformation. Some mathematical models in the field of nanotechnology are contained, for example, in articles [1,2] (see also references in them). In mathematical modeling of processes taking place in viscoelastic materials, there is a so-called system with memory, whose behavior is not completely determined by the state at the moment, but depends on the systems entire history, and therefore, describes an integro-differential equation that contains the corresponding integral with respect to the time variable. The system of equations (1), taking into account the integral term (3) is the basic in the linear theory of viscoelastic anisotropic media.

A study of inverse problems for hyperbolic integro-differential equations and systems is the subject of research by many authors. Among the problems that are closer to the present work can be identified [3-9]. In papers [3,4], the unique solvability and stability of the solution for the inverse problem for the identification of a memory kernel from Maxwell's system integro-differential equations for a homogeneous anisotropic media are studied. In 
work [5], the inverse problem for a second order hyperbolic equation with an integral member of convolution type with respect to one-dimensional time - dependent memory function of the medium and solution of the direct problem is investigated. By Fourier's method, this problem is reduced to solving the Volterra integral equations with respect to the unknown functions of the time-dependent variable. In papers [6,7] (see also references therein) the problem of determining the multidimensional kernel in viscoelasticity equation for an inhomogeneous isotropic medium is investigated. In [8,9], the problem of the one-dimensional kernel reconstruction from viscoelasticity equation in the bounded and unbounded domains has been studied. The theorems for the global unique solvability of these problems in the class of continuous functions with weighted norms were proved. The basic feature inherent in [3, 4, 6-9] and this paper is to use a boundary-localized and/or a fixed point of the spatial domain source, for the initiation of the physical process of wave propagation. Finally, we recall that the papers [10-13] are concerned with the problems of kernel determination from integro-differential equations with an integral of the convolution type. In the present paper, the approach of the works $[3,4]$ will be used.

We will consider the problem $(1)-(2)$ for the case in which the function $f(x, t)$ has the form:

$$
f(x, t)=\vec{e} \delta(x) \delta^{\prime}(t)
$$

where $\vec{e}=\left(\frac{1}{\sqrt{3}}, \frac{1}{\sqrt{3}}, \frac{1}{\sqrt{3}}\right)$ is unit vector; $\delta(x)=\delta\left(x_{1}\right) \delta\left(x_{2}\right) \delta\left(x_{3}\right)$ is the Dirac delta function of the space variable concentrated at $x_{1}=0, x_{2}=0, x_{3}=0 ; \delta^{\prime}(t)$ is the derivative of Dirac delta function of the time variable concentrated at $t=0$.

The problem in which the vector $u(x, t)=\left(u_{1}, u_{2}, u_{3}\right)(x, t)$ should be determined from (1) - (4) for given matrices $K(t)=\operatorname{diag}\left(K_{1}, K_{2}, K_{3}\right)(t), C=\left(c_{\alpha \beta}\right)_{(6 \times 6)}$ and number $\rho>0$ will be called the direct problem.

Let $U(\nu, t)=\left(U_{1}, U_{2}, U_{3}\right)(\nu, t)$ be the Fourier image of $u(x, t)$ with respect to $x=\left(x_{1}, x_{2}, x_{3}\right) \in R^{3}$, respectively, i.e.:

$$
U_{j}(\nu, t)=\int_{R} u_{j}(x, t) e^{i(x, \nu)} d x, \quad \nu=\left(\nu_{1}, \nu_{2}, \nu_{3}\right) \in R^{3}, \quad(x, \nu)=\sum_{\lambda=1}^{3} x_{\lambda} \nu_{\lambda}, \quad j=1,2,3,
$$

where $\nu$ is the parameter of transformation. We pose the following inverse problem: find the matrix function $K(t)=\operatorname{diag}\left(K_{1}, K_{2}, K_{3}\right)(t), t \geq 0$ occurring in the integral in equations (3) from the information on the Fourier image $U(\nu, t)$ at an arbitrary time $t \geq 0$ for the values $\nu=\nu_{0}$ of the Fourier transformation:

$$
U\left(\nu_{0}, t\right)=g(t), \quad g(t)=\left(g_{1}, g_{2}, g_{3}\right)
$$

Definition. A solution of the inverse problem is a matrix function $K(t)=\operatorname{diag}\left(K_{1}, K_{2}, K_{3}\right)(t)$ such that the corresponding solution of problem (1) - (4) satisfies condition (5).

The main results of the present paper are the following theorems:

Theorem 1. Let us fix some arbitrary $T, T>0$. Suppose that $g(t) \in C^{3}[0, T]$ and the agreement conditions:

$$
g(0)=\frac{\vec{e}}{\rho}, \quad g^{\prime}(0)=0, \quad g^{\prime \prime}(0)=\frac{1}{\rho^{2}} Q\left(\nu_{0}\right) \vec{e}
$$

holds. Besides, $\operatorname{det} Q\left(\nu_{0}\right) \neq 0, \nu_{0} \neq 0$, where $Q(\nu)$ is matrix definite by formula (12). Then, the inverse problem (1) - (5) has a unique solution $K(t)=\operatorname{diag}\left(K_{1}, K_{2}, K_{3}\right)(t) \in C[0, T]$.

Let $G(\gamma)$ be the set of functions $g(t)$, satisfying the conditions of theorem 1 and $\left\|g_{i}(t)\right\|_{C^{3}[0, T]} \leq \gamma<\infty$, $t \in[0, T], i=1,2,3, \gamma-$ the given number.

Theorem 2. Let $K^{m}(t)=\operatorname{diag}\left(K_{1}^{m}(t), K_{2}^{m}(t), K_{3}^{m}(t)\right)$ be solution to the inverse problem (1) - (5) with $g^{m}(t) \in G(\gamma), m=1,2$, respectively. Then, there exists positive constant $C$, depending on numbers T, $\rho, \gamma$ and elements of matrix $Q\left(\nu_{0}\right)$ so that the following estimate of stability is valid:

$$
\sum_{i=1}^{3}\left\|K_{i}^{1}-K_{i}^{2}\right\|_{C[0, T]} \leq C \sum_{i=1}^{3}\left\|g_{i}^{1}-g_{i}^{2}\right\|_{C^{3}[0, T]}
$$




\section{The main equations with respect to Fourier's image}

Denote

$$
\bar{T}_{i j}:=\sum_{k, l=1}^{3} c_{i j k l} \frac{\partial v_{k}}{\partial x_{l}}(x, t)
$$

Then, (3) can be written as the following:

$$
T_{i j}(x, t)=\bar{T}_{i j}(x, t)+\int_{0}^{t} K_{i}(\tau) \bar{T}_{i j}(x, t-\tau) d \tau, \quad i=1,2,3 ; j=1,2,3 .
$$

Using the symmetry properties of the elastic moduli and the rule of the renumbering of indices, accepted above, relation (7) obtains the form:

$$
\begin{array}{r}
\bar{T}_{\alpha}=c_{\alpha 1} \frac{\partial v_{1}}{\partial x_{1}}+c_{\alpha 6} \frac{\partial v_{1}}{\partial x_{2}}+c_{\alpha 5} \frac{\partial v_{1}}{\partial x_{3}}+c_{\alpha 6} \frac{\partial v_{2}}{\partial x_{1}}+c_{\alpha 2} \frac{\partial v_{2}}{\partial x_{2}}+c_{\alpha 4} \frac{\partial v_{2}}{\partial x_{3}}+c_{\alpha 5} \frac{\partial v_{3}}{\partial x_{1}}+c_{\alpha 4} \frac{\partial v_{3}}{\partial x_{2}}+c_{\alpha 3} \frac{\partial v_{3}}{\partial x_{3}} \\
\alpha=1,2, \ldots, 6
\end{array}
$$

Therefore, for each $i=1,2,3$, the first terms on the right side of (1) can be written as:

$$
\begin{aligned}
& \sum_{j=1}^{3} \frac{\partial \bar{T}_{1 j}}{\partial x_{j}}=\frac{\partial \bar{T}_{1}}{\partial x_{1}}+\frac{\partial \bar{T}_{6}}{\partial x_{2}}+\frac{\partial \bar{T}_{5}}{\partial x_{3}} \\
& \sum_{j=1}^{3} \frac{\partial \bar{T}_{2 j}}{\partial x_{j}}=\frac{\partial \bar{T}_{6}}{\partial x_{1}}+\frac{\partial \bar{T}_{2}}{\partial x_{2}}+\frac{\partial \bar{T}_{4}}{\partial x_{3}} \\
& \sum_{j=1}^{3} \frac{\partial \bar{T}_{3 j}}{\partial x_{j}}=\frac{\partial \bar{T}_{5}}{\partial x_{1}}+\frac{\partial \bar{T}_{4}}{\partial x_{2}}+\frac{\partial \bar{T}_{3}}{\partial x_{3}}
\end{aligned}
$$

Formulae (8) and (9) will be used for computing the Fourier's transformation with respect to the variable $x$ of the right sides in (1).

We apply the Fourier transformation to both parts of (1) - (4). The Fourier transform of the vector function $u(x, t)$ exists at any finite $t$, since the vector function $u(x, t)$, as the solution of the direct problem (1) - (4), is the sum of a certain singular generalized vector function and a regular vector function, the support of the vector function $u(x, t)$ being finite [14, chapter 4]. At any fixed $\nu$, the vector function $U(\nu, t), U(\nu, t)-$ the Fourier transformation of $u(x, t)$ with respect to $x$ satisfies differential equation:

$$
\rho I \frac{\partial^{2} U}{\partial t^{2}}=Q(\nu) U(\nu, t)+\int_{0}^{t} K(t-\tau) Q(\nu) U(\nu, \tau) d \tau+\vec{e} \delta^{\prime}(t), \quad \nu \in R^{3}, t \in R
$$

for the initial conditions:

$$
\left.\tilde{V}\right|_{t \leq 0}=0
$$

In equation (10), we denoted $I-$ the unit matrix of third order, $U(\nu, t)=\left(U_{1}, U_{2}, U_{3}\right)^{*}(\nu, t), \vec{e}=\left(e_{1}, e_{2}, e_{3}\right)=$ $\left(\frac{1}{\sqrt{3}}, \frac{1}{\sqrt{3}}, \frac{1}{\sqrt{3}}\right)$

$$
Q(\nu)=\left(\begin{array}{lll}
Q_{11} & Q_{12} & Q_{13} \\
Q_{21} & Q_{22} & Q_{23} \\
Q_{31} & Q_{32} & Q_{33}
\end{array}\right),
$$

$Q_{i j}(\nu), 1 \leq i \leq 3,1 \leq j \leq 3-$ uniform polynomials of second order with respect to $\nu$ : 


$$
\begin{aligned}
& Q_{11}(\nu)=c_{11} \nu^{2}+2 c_{16} \nu_{1} \nu_{2}+c_{66} \nu_{2}^{2}+2 c_{15} \nu_{1} \nu_{3}+2 c_{56} \nu_{2} \nu_{3}+c_{55} \nu_{3}^{2} ; \\
& Q_{12}(\nu)=c_{16} \nu_{1}^{2}+\left(c_{12}+c_{66}\right) \nu_{1} \nu_{2}+c_{62} \nu_{2}^{2}+\left(c_{14}+c_{56}\right) \nu_{1} \nu_{3}+\left(c_{52}+c_{64}\right) \nu_{2} \nu_{3}+c_{54} \nu_{3}^{2} ; \\
& Q_{13}(\nu)=c_{15} \nu_{1}^{2}+\left(c_{14}+c_{65}\right) \nu_{1} \nu_{2}+c_{64} \nu_{2}^{2}+\left(c_{13}+c_{55}\right) \nu_{1} \nu_{3}+\left(c_{63}+c_{54}\right) \nu_{2} \nu_{3}+c_{53} \nu_{3}^{2} ; \\
& Q_{21}(\nu)=c_{61} \nu_{1}^{2}+\left(c_{21}+c_{66}\right) \nu_{1} \nu_{2}+c_{26} \nu_{2}^{2}+\left(c_{41}+c_{65}\right) \nu_{1} \nu_{3}+\left(c_{25}+c_{46}\right) \nu_{2} \nu_{3}+c_{45} \nu_{3}^{2} ; \\
& Q_{22}(\nu)=c_{66} \nu_{1}^{2}+2 c_{26} \nu_{1} \nu_{2}+c_{22} \nu_{2}^{2}+2 c_{64} \nu_{1} \nu_{3}+2 c_{24} \nu_{2} \nu_{3}+c_{44} \nu_{3}^{2} ; \\
& Q_{23}(\nu)=c_{65} \nu_{1}^{2}+\left(c_{64}+c_{25}\right) \nu_{1} \nu_{2}+c_{24} \nu_{2}^{2}+\left(c_{45}+c_{63}\right) \nu_{1} \nu_{3}+\left(c_{23}+c_{44}\right) \nu_{2} \nu_{3}+c_{43} \nu_{3}^{2} ; \\
& Q_{31}(\nu)=c_{51} \nu_{1}^{2}+\left(c_{41}+c_{56}\right) \nu_{1} \nu_{2}+c_{46} \nu_{2}^{2}+\left(c_{31}+c_{55}\right) \nu_{1} \nu_{3}+\left(c_{36}+c_{45}\right) \nu_{2} \nu_{3}+c_{35} \nu_{3}^{2} ; \\
& Q_{32}(\nu)=c_{56} \nu_{1}^{2}+\left(c_{46}+c_{52}\right) \nu_{1} \nu_{2}+c_{42} \nu_{2}^{2}+\left(c_{54}+c_{36}\right) \nu_{1} \nu_{3}+\left(c_{32}+c_{44}\right) \nu_{2} \nu_{3}+c_{34} \nu_{3}^{2} ; \\
& Q_{33}(\nu)=c_{55} \nu_{1}^{2}+2 c_{45} \nu_{1} \nu_{2}+c_{44} \nu_{2}^{2}+2 c_{35} \nu_{1} \nu_{3}+2 c_{34} \nu_{2} \nu_{3}+c_{33} \nu_{3}^{2},
\end{aligned}
$$

Thus, the inverse problem (1) - (4) is reduced to the problem of determining the kernel $K(t), t>0$ of the integral part in the equation (10) on the bases of equalities (10), (11) and (5).

\section{Proof of the main results}

Taking into consideration the fact that $t \theta(t)$, where $\theta(t)$ - the Heavyside step function, is the fundamental solution of differential operator $\partial^{2} / \partial t^{2}$, the solution to the direct problem (10), (11) can be represented in the form:

$$
\begin{aligned}
U(\nu, t)=\int_{R}(t-\tau) \theta(t-\tau) & {\left[\frac{1}{\rho} Q(\nu)\left(U(\nu, t)+\int_{0}^{\tau} K(\alpha) U(\nu, \tau-\alpha) d \alpha\right)+\frac{\vec{e}}{\rho} \delta^{\prime}(\tau)\right] d \tau } \\
= & \theta(t)\left[\frac{\vec{e}}{\rho}+\frac{1}{\rho} \int_{0}^{t}(t-\tau) Q(\nu)\left(U(\nu, t)+\int_{0}^{\tau} K(\alpha) U(\nu, \tau-\alpha) d \alpha\right) d \tau\right] .
\end{aligned}
$$

To prove Theorem 1, we begin by setting $\nu=\nu_{0}$ in this equation and using supplementary condition (5). This results in the following equality:

$$
g(t)=\frac{\vec{e}}{\rho}+\frac{1}{\rho} \int_{0}^{t}(t-\tau) Q\left(\nu_{0}\right)\left(g(t)+\int_{0}^{\tau} K(\alpha) g(\tau-\alpha) d \alpha\right) d \tau .
$$

Differentiating equation (13) twice with respect to the variable $t$, we have the following equalities:

$$
\begin{aligned}
& g^{\prime}(t)=\frac{Q\left(\nu_{0}\right)}{\rho}\left[\int_{0}^{t} g(\tau) d \tau+\int_{0}^{t} \int_{0}^{\tau} K(\alpha) g(\tau-\alpha) d \alpha d \tau\right], \\
& g^{\prime \prime}(t)=\frac{Q\left(\nu_{0}\right)}{\rho} g(t)+\frac{Q\left(\nu_{0}\right)}{\rho} \int_{0}^{t} K(\alpha) g(t-\alpha) d \alpha .
\end{aligned}
$$

To obtain an integral equation for $K(t)$, we differentiate the last relation again with respect to $t$. Multiplying the left-hand side of result equality by $Q^{-1}\left(\nu_{0}\right)$, after easily transforming, one gets:

$$
K(t)=\sqrt{3} \rho^{2} Q^{-1}\left(\nu_{0}\right) g^{\prime \prime \prime}(t)-\sqrt{3} \rho g^{\prime}(t)-\sqrt{3} \rho \int_{0}^{t} K(\alpha) g^{\prime}(t-\alpha) d \alpha .
$$

For each fixed $i(14)$, is a linear integral second-order equation with respect to the unknown vector function $K(t)$. As is known, these equations have unique solutions.

We now prove Theorem 2. Consider the integral equation (14) for each $i$ for $g^{m}(t), m=1,2$. Corresponding to these functions, the solutions of the integral equation (14) are denoted by $K_{i}^{m}(t), m=1,2$, making the difference $K_{i}^{1}(t)-K_{i}^{2}(t)$, from which we estimate it. From (14), one gets: 


$$
\left|K_{i}^{1}(t)-K_{i}^{2}(t)\right| \leq C_{1}\left\|g_{i}^{1}-g_{i}^{2}\right\|_{C^{3}[0, T]}+C_{2} \int_{0}^{t}\left|K_{i}^{1}(\tau)-K_{i}^{2}(\tau)\right| d \tau, \quad i=1,2,3 .
$$

Therefore, taking into account Gronwall's inequality, we have:

$$
\left|K_{i}^{1}(t)-K_{i}^{2}(t)\right| \leq C_{3}\left\|g_{i}^{1}-g_{i}^{2}\right\|_{C^{3}[0, T]}, \quad i=1,2,3 .
$$

Constants $C_{j}, j=1,2,3$ depend on the numbers $T, \rho, \gamma$ and elements of matrix $Q\left(\nu_{0}\right)$. The last equalities yield estimate (6).

\section{References}

[1] Dvurechenskii A., Alfimov M., et al. IV Nanotechnology International Forum (RUSNANOTECH 2011). J. Phys.: Conf. Series, 2012, 345 (1), 011001

[2] Chivilikhin S.A., Gusarov V.V., Popov I.Yu. Flows in nanostructures: hybrid classical-quantum models. Nanosystems: Physics, Chemistry, Mathemetics, 2012, 3 (1), P. 7-26.

[3] Durdiev D.K. Inverse problem for the identification of a memory kernel from Maxwell's system integro-differential equations for a homogeneous anisotropic media. Nanosystems: Physics, Chemistry, Mathematics, 2015, 6 (2), P. $268-273$.

[4] Durdiev D.K., Durdiev U.D. Stability of the inverse problem solution for Maxwell's system integro-differential equations in a homogeneous anisotropic media. Uzbek Mathematical Journal, 2014, 2, P. 25-34. (in Russian)

[5] Janno J., Von Welfersdorf L. Inverse problems for identification of memory kernels in viscoelasticity. Math. Methods in Appl. Sciences, 1997, 20 (4), P. 291-314.

[6] Romanov V.G. Stability estimates for the solution in the problem of determining the kernel of the viscoelasticity equation. Sib. Zh. Ind. Mat., 2012, 15 (1), P. 86-98. (in Russian)

[7] Durdiev D.K., Totieva Zh.D. The problem of determining the multidimentional kernel of viscoelasticity equation. Vladikavkaz. Mat. Zh., 2015, 17 (4), P. 18-43. (in Russian)

[8] Durdiev D.K., Safarov Zh.Sh. Inverse problem of determining the one-dimensional kernel of the viscoelasticity equation in a bounded domain. Mat. Zametki, 2015, 97 (6), P. 855-867. (in Russian)

[9] Durdiev D.K., Totieva Zh.D. The problem of determining the one-dimensional kernal of the viscoelasticity equation. Sib. Zh. Ind. Mat., 2013, 16 (2), P. 72-82. (in Russian)

[10] Jaan J., Von Wolfersdorf L. An inverse problem for identification of a time- and space-dependent memory kernel in viscoelasticity. Inverse Problems, 2001, 17, P. 13-24.

[11] Colombo F., Guidetti D. A global in time existence and uniqueness result for a semilinear integrodifferential parabolic inverse problem in Sobolev spaces. Math. Methods Appl. Sciences, 2007, 17, P. 1-29.

[12] Colombo F., Guidetti D. Some results on the Identification of memory kernels. Oper. Theory: Adv. Appl., 2011,216, P. $121-138$.

[13] Favaron A. Identification of Memory Kernels Depending on Time and on an Angular Variable. Zeitschrift fur Analysis und ihre Anwendungen, 2005, 24 (4), P. 735-762.

[14] Romanov V.G. Inverse problems of mathematical physics. Nauka, Moscow, 1984. (in Russian) 